DOI 10.18699/SBB-2020-35

\title{
Genes determining dwarfism in Cucurbitaceae
}

Strygina K.*, Tekhanovich G., Elatskova A., Elatskov Yu., Khlestkina E.

Federal Research Center N. I. Vavilov All-Russian Institute of Plant Genetic Resources, Saint Petersburg, Russia

*e-mail: k.strygina@vir.nw.ru

Key words: Citrullus, Cucumis, Cucurbita, dwarf genes, short vine

Motivation and Aim: The melon crops (family Cucurbitaceae), such as pumpkin (genus Cucurbita), muskmelon (genus Cucumis), and watermelon (genus Citrullus) are both heat and light preferring plants. These crops are widely distributed on the territory of Russia and are cultivated as food and feed plants. Using methods of molecular genetics in combination with marker-assisted selection, it is possible to create dwarf varieties growing in more northern latitudes, including beyond the Arctic Circle. The aim of this work is to identify and mark the genes responsible for the formation of dwarf plant forms based on the unique collection of melon crops of the Federal Research Center N. I. Vavilov All-Russian Institute of Plant Genetic Resources (VIR).

Methods and Algorithms: The search of homologous sequences was made in CuGenDB database BLASTN. The cluster analysis using the MEGA software was based on NJ algorithm. Development of diagnostic primers for the alleles determination as well as primers for the PCR and sequencing was performed using the OLIGO program. A total of 13 C. lanatus varieties were studied.

Results: In genomes of watermelon C. lanatus, cucumber $C$. sativus, muskmelon $C$. melo, pumpkins and squashes C. pepo, C. moschata, C. maxima, C. argyrosperma, wax gourd Benincasa hispida, bottle gourd Lagenaria siceraria the search of homologous sequences of watermelon dwarf genes Cla010337 (ABC transporter), Cla010726 (gibberellin 20-oxidase) and Cla015407 (gibberellin 3-beta-hydroxylase) was performed. In genomes of analyzed organisms one copy of Cla010337, one copy of Cla010726 and two copies of Cla015407 were identified. Time of occurrence of each new copy is calculated and presented in the report. The allelic polymorphism was analyzed in genomes of VIR watermelon varieties.

Conclusion: All available sequences have conserved domains. Significant change in the motives, which could lead to changes in gene function, has been identified during sequencing in varieties with short vine. Acknowledgements: The present study was supported by the VIR project No. 0481-2019-0001. 